\title{
ACR TIRADS is Best to Decrease the Number of Thyroid Biopsies and Maintain Accuracy
}

\section{Stephanie A. Fish}

Department of Endocrinology, Memorial Sloan Kettering Cancer Center, New York, New York, U.S.A.

Review of: Grani G, Lamartina L, Ascoli V, Bosco D, Biffoni M, Giacomelli L, Maranghi M, Falcone R, Ramundo V, Cantisani V, Filetti S, Durante C 2019 Reducing the number of unnecessary thyroid biopsies while improving diagnostic accuracy: toward the "right" TIRADS. J Clin Endocrinol Metab 104:95-102. PMID: 30299457.

\section{SUMMARY}

\section{Background}

Thyroid nodules are a common finding, especially due to the increased utilization of imaging studies of the neck. Most thyroid nodules are benign. The key in evaluating thyroid nodules is to identify the few nodules that contain a clinically relevant malignancy. Fine-needle aspiration (FNA) is the test of choice to evaluate nodules for evidence of malignancy. However, at least half of all thyroid biopsy results are benign (1) and up to one third of biopsies yield indeterminate cytologic results (2). Ultrasound plays a key role in identifying those nodules most at risk for thyroid cancer. Several ultrasound-based risk stratification systems have now been developed to determine which nodules should be referred for biopsy (3-7). However, there are few data comparing the different guidelines to determine which is best at identifying thyroid cancer while minimizing the number of thyroid biopsies.

This is a prospective, observational study of 502 thyroid nodules referred to a single center for FNA (8). Each nodule was classified based on the ultrasound features using the five risk-stratification systems. Then, the recommendation for FNA was evaluated as it related to the nodule's pathologic diagnosis. The aims of the study were to determine the number of FNAs that would have been consid- ered unnecessary by each system and the reliability of these exclusions.

\section{Methods}

All patients referred to the thyroid cancer unit of a large academic referral center for FNA of a thyroid nodule between November 2015 and May 2018 were considered for the study. Prior to the biopsy, each nodule was examined with a HI VISION Avius ultrasound system and a $13-\mathrm{MHz}$ linear-array transducer. Two experienced clinicians recorded a consensus assessment of the sonographic features of each nodule. The nodule features evaluated included size, margins, structure, echogenicity, calcifications, other hyperechoic foci, and extrathyroidal extension. The shape was considered taller than wide when the anterior-posterior diameter exceeded the transverse diameter.

For each nodule, the determination of each ultrasound feature was used to classify the risk of malignancy according to five ultrasound-based risk-stratification systems (ATA, AACE/ACE/AME, ACR TIRADS, EU-TIRADS, and K-TIRADS) (3-7). Within each risk classification, FNA is recommended based on the size of the nodule. The investigators identified the nodules for which FNA would be recommended based on the size threshold for the assigned risk 

Number of Thyroid Biopsies and Maintain Accuracy

class for each of the five risk-stratification systems. Since none of the systems recommend FNA of subcentimeter nodules, all nodules less than $1 \mathrm{~cm}$ were excluded from the study.

The biopsies were done under ultrasound guidance by two clinicians. Direct smears were analyzed by experienced thyroid cytopathologists and classified according to the criteria published in the Italian Consensus for Thyroid Cytopathology $(9,10)$. When surgery was performed, the final diagnosis was based on the surgical pathology. When the nodule was managed without surgery, the final diagnosis was based on the FNA cytology. Nodules with nondiagnostic or indeterminate cytology were excluded from the final analysis.

Biopsies ordered for nodules that did not meet criteria for FNA based on the risk-stratification system were considered "unnecessary." The guideline recommendation regarding FNA was then compared with the final nodule diagnosis (benign vs. malignant) to estimate the sensitivity, specificity, positive predictive value, negative predictive value, diagnostic odds ratio, and area under the receiver-operating-characteristic curve.

\section{Results}

A total of 832 nodules were evaluated with ultrasound prior to FNA. Seventy-nine nodules measured less than $1 \mathrm{~cm}$ and were excluded from the study. Another 251 nodules were excluded owing to inde- terminate cytology results. The final cohort included 502 nodules in 477 patients (mean $[ \pm$ SD] age, $55.9 \pm 13.9$ years). Thirty-six $(7.2 \%)$ nodules were determined to be malignant.

In general, strictly adhering to the recommendations from the risk-stratification systems reduced the number of FNAs to between $17.1 \%$ and $53.4 \%$. The most effective system was the ACR TIRADS, which would have reduced the number of biopsies by more than half $(53.4 \%)$ with a false negative rate of $2.2 \%$. The false negative rate was due to nodules with a final diagnosis of malignancy, but no biopsy recommendation based on the risk-stratification system. Most of the systems had similar discriminatory capacities to identify malignancy, but they recommended completing many more biopsies. K-TIRADS was the weakest performer, as it reduced the number of biopsies by only $17.1 \%$. Eleven nodules diagnosed as malignant would have been misclassified as not requiring FNA by at least one of the TIRADS systems. Three cancers were missed by all five systems; these were either isoechoic or hyperechoic and had no other features considered suspicious.

\section{Conclusions}

Ultrasound-based risk-stratification systems vary widely in their ability to reduce the number of unnecessary thyroid nodule FNAs. The ACR TIRADS performed best by classifying more than half of FNAs as unnecessary with a false negative rate of only $2.2 \%$.

\section{COMMENTARY}

Determining the most appropriate and efficient evaluation system for thyroid nodules is challenging for a clinician. While most nodules are benign, it is important to identify those nodules at highest risk for malignancy in order to recommend FNA. Ultrasound features are helpful in identifying high-risk nodules. However, there are five different ultrasound-based risk-stratification systems available to evaluate nodules. There are few studies that directly compare the different systems, and those that do are retrospective $(11,12)$. To best address this common clinical problem, it is important to identify the system 

Number of Thyroid Biopsies and Maintain Accuracy

that recommends the fewest FNAs but identifies the vast majority of malignancies.

The goal of this study was to compare the performances of the five most widely used ultrasound-based risk-stratification systems to determine which system is best at identifying nodules at low risk of malignancy that do not need further evaluation with FNA (8). The authors found that the number of biopsies would have been reduced if any of the systems had been used consistently. However, the most significant reduction in FNAs was seen when using the ACR TIRADS system. Despite recommending less than half of the FNAs, very few malignant nodules were missed with this system.

The strengths of the study include the prospective nature and the large cohort. In addition, the ultrasound feature assessment was completed in real time prior to the FNA. The clinicians performing the ultrasounds and cytopathologists evaluating the FNA samples were experts in the fields, ensuring the accuracy of the results.

However, there are limitations to the study. The initial cohort was a selected group of patients referred for FNA by an outside clinician and therefore may not reflect the general population. In addition, the authors excluded nodules with nondiagnostic and indeterminate cytology. So one cannot make conclusions about the accuracy of the risk-stratifica- tion systems for these nodules. Finally, there were surgical pathology results-the gold standard for thyroid cancer diagnosis-in only a select group of nodules whose cytology showed malignancy. For the majority of the nodules, the cytologic diagnosis was used as the final diagnosis. It is possible that there were some false negative cytology results.

Despite these limitations, these results are clinically important, as they indicate that ACR TIRADS is the best ultrasound-based risk-stratification system for evaluating thyroid nodules. Based on these results, it seems that this system should be used more universally. However, for any of these systems to be beneficial, they need to be applied accurately. This has been a clinical challenge, as there can be significant interobserver variability in the interpretation of the ultrasonographic features of thyroid nodules. In addition, the ACR TIRADS has a point system for each ultrasound feature and the sum of the points determines the risk class. The other systems rely on pattern recognition. Thus, the ACR TIRADS can be a bit more time-consuming to implement. For this system to be beneficial to patients with thyroid nodules, continued training of radiologists and endocrinologists is important so that the system can be utilized accurately. With precise implementation, it appears that ACR TIRADS will provide the most efficient and accurate care for the many patients with thyroid nodules.

\section{References}

1. Bongiovanni M, Spitale A, Faquin WC, Mazzucchelli L, Baloch ZW 2012 The Bethesda System for Reporting Thyroid Cytopathology: a meta-analysis. Acta Cytol 56:333-339. Epub 2012 Jul 25.

2. Durante C, Grani G, Lamartina L, Filetti S, Mandel SJ, Cooper DS 2018 The diagnosis and management of thyroid nodules: a review. JAMA 319:914-924.
3. Haugen BR, Alexander EK, Bible KC, Doherty GM, Mandel SJ, Nikiforov YE, Pacini F, Randolph GW, Sawka AM, Schlumberger M, et al. 2016 2015 American Thyroid Association management guidelines for adult patients with thyroid nodules and differentiated thyroid cancer: the American Thyroid Association Guidelines Task Force on 

Number of Thyroid Biopsies and Maintain Accuracy

Thyroid Nodules and Differentiated Thyroid Cancer. Thyroid 26:1-133.

4. Gharib H, Papini E, Garber JR, Duick DS, Harrell RM, Hegedus L, Paschke R, Valcavi R, Vitti P 2016 American Association of Clinical Endocrinologists, American College of Endocrinology, and Associazione Medici Endocrinologi medical guidelines for clinical practice for the diagnosis and management of thyroid nodules-2016 update. Endocr Pract 22:622-639.

5. Russ G, Bonnema SJ, Erdogan MF, Durante C, Ngu R, Leenhardt L 2017 European Thyroid Association guidelines for ultrasound malignancy risk stratification of thyroid nodules in adults: the EUTIRADS. Eur Thyroid J 6:225-237. Epub 2017 Aug 8.

6. Shin JH, Baek JH, Chung J, Ha EJ, Kim JH, Lee YH, Lim HK, Moon WJ, Na DG, Park JS, et al. 2016 Ultrasonography diagnosis and imaging-based management of thyroid nodules: revised Korean Society of Thyroid Radiology consensus statement and recommendations. Korean J Radiol 17:370-395. Epub 2016 Apr 14.

7. Tessler FN, Middleton WD, Grant EG, Hoang JK, Berland LL, Teefey SA, Cronan JJ, Beland MD, Desser TS, Frates MC, et al. 2017 ACR Thyroid Imaging, Reporting and Data System (TI-RADS): White paper of the ACR TI-RADS committee. J Am Coll Radiol 14:587-595. Epub 2017 Apr 2.
8. Grani G, Lamartina L, Ascoli V, Bosco D, Biffoni M, Giacomelli L, Maranghi M, Falcone R, Ramundo V, Cantisani V, et al. 2019 Reducing the number of unnecessary thyroid biopsies while improving diagnostic accuracy: toward the "right" TIRADS. J Clin Endocrinol Metab 104:95-102.

9. Nardi F, Basolo F, Crescenzi A, Fadda G, Frasoldati A, Orlandi F, Palombini L, Papini E, Zini M, Pontecorvi A, Vitti P 2014 Italian consensus for the classification and reporting of thyroid cytology. J Endocrinol Invest 37:593-599. Epub 2014 May 1.

10. Fadda G, Basolo F, Bondi A, Bussolati G, Crescenzi A, Nappi O, Nardi F, Papotti M, Taddei G, Palombini L 2010 Cytological classification of thyroid nodules. Pathologica 102:405-408.

11. Yoon JH, Lee HS, Kim EK, Moon HJ, Kwak JY 2016 Malignancy risk stratification of thyroid nodules: comparison between the Thyroid Imaging Reporting and Data System and the 2014 American Thyroid Association management guidelines Radiology 278:917-924. Epub 2015 Sep 8.

12. Middleton WD, Teefey SA, Reading CC, Langer JE, Beland MD, Szabunio MM, Desser TS 2018 Comparison of performance characteristics of American College of Radiology TI-RADS, Korean Society of Thyroid Radiology TIRADS, and American Thyroid Association guidelines. AJR Am J Roentgenol 210:1148-1154. Epub 2017 Apr 12. 